\title{
Protocol: How Has Sustainable Development Goals Declaration Influenced Health Financing Reform Processes for Universal Health Coverage at the Country Level? A Systematic Review of Literature
}

\section{Walter Denis Odoch ( $\nabla$ wodoch2018@gmail.com )}

African Centre for Health Systems Development https://orcid.org/0000-0003-0376-4594

Flavia Senkubuge

University of Pretoria School of Health Systems and Public Health

Charles Hongoro

Human Sciences Research Council of South Africa

\section{Study Protocol}

Keywords: Sustainable Development Goals, Universal Health Coverage, Health Financing

Posted Date: October 21st, 2020

DOI: https://doi.org/10.21203/rs.3.rs-93291/v1

License: (c) (i) This work is licensed under a Creative Commons Attribution 4.0 International License.

Read Full License 


\section{Abstract}

\section{Background}

Achieving universal health coverage (UHC) is necessitating reforms in the way countries finance health systems. However, health financing reforms (HFR) are inherently political and SDGs declaration is a political one. Therefore, the terrain where efforts for reforming health financing occurs is likely being influenced by the SDGs declaration. However, how the SDGs have influenced health financing reform processes at the national level, the outcomes of these processes and how stakeholders especially ministries of health are using SDGs to influence reforms towards UHC have not been explored. This review is proposed to provide information and lessons on how SDGs have influence health financing reforms for UHC at national level and identify methodological and empirical gaps in studies exploring the relationship between SDGs and health financing reforms for UHC.

Methods

We propose a systematic review of literature and shall adhere to preferred reporting items for systematic review and meta-analysis (PRISMA) guidelines. We shall search 5 databases (Ovid Medline, PubMed, EBSCO, Scopus and Web of Science) and grey literature. Boolean operators "OR" and "AND" will be used to combine the concepts of sustainable development goals and health financing. The literature to be reviewed will be those on health financing reforms (population), one of the factors influencing the reform is SDGs declaration (exposure/intervention), and there is change in a health financing function (outcome). Identification and selection of studies shall be done in duplicate using a predefined tool, the overall process shall be depicted in a PRISMA flow chart. Quality assessment will be conducted using Joanna Biggs Institute checklist. Data shall be managed in NVIVo, where a thematic synthesis of data will be conducted. The results will be presented narratively.

\section{Discussion}

We shall share our finding with relevant stakeholders through workshop and publications. The SDGs declaration is relatively new, how it is influencing health financing reforms towards $\mathrm{UHC}$ at national level needs to be documented and shared for cross-country learning by stakeholders involved in this area. In addition, the review is anticipated to provide information on the methodological and/or empirical gaps in studies exploring the linkage between SDGs declaration and health systems reforms.

\section{Background}

Global declarations shape public policy priorities and guide development finance flows (1-3). The declarations usually energize governmental processes and provide reference point that guides national policies toward priority development issues. Therefore, global and regional declarations can be powerful tools for shaping policy and programme response on issues that are of public concern (4). In September 2015 in New York, the United Nations (UN) member states leaders, meeting under the theme, 
"Transforming our world: the 2030 Agenda for Sustainable Development", adopted 17 sustainable development goals (SDGs) as the post millennium development goals (MDGs) development blue print (5). Health is explicitly addressed in paragraph 26 of the SDGs declaration (6):

To promote physical and mental health and well-being, and to extend life expectancy for all, we must achieve universal health coverage and access to quality health care. No one must be left behind...

Achieving universal health coverage (UHC) is one of the overarching targets of the 2030 agenda for sustainable development. Universal health coverage is based on the precept that all people should have access to health services they need and do not suffer financial hardship while accessing the services (7). This implies, an effective, efficient and equitable health financing system is a critical and essential component that contributes to achievement of UHC target under the SDGs declaration (8-11). Health financing influences progress on the three UHC goals of equity in the use of health services, quality of care and financial protection through effects on UHC intermediary objectives of transparency and accountability, efficiency and equity in resource distribution $(8,12)$. Therefore, health financing is central to efforts to improve health and health systems, because it is only when resources are adequately, efficiently and equitably mobilized, pooled and spent that all people can enjoy sustained progress towards UHC $(13,14)$.

For many countries achievement of UHC require reforms in health financing system so that people have financial protection while accessing quality health care (15). Therefore, a number of countries in the African region have been attempting to reform the way health is financed to ensure sustainable progress towards UHC (15-19). However, these attempts, largely spearheaded by Ministries of Health have been slow and interruptive as reflected in the slow and/or forward and backward movements in the reform processes $(16,20-22)$. The process of introducing comprehensive National Health Insurance Schemes (NHIS), has been ongoing in the last 2 to 3 decades in Uganda, Kenya, Zambia and South Africa $(16,20-$ 24). In South Africa, Uganda, Kenya and Zambia Health Insurance Bills were (or have been) drafted, however they have not reached parliament or where the parliament approved the Bills, they never became Acts due to failure by the Executive arm of governments to ascent to the Bills or the process took very long $(22,25,26)$.

The terrain where various efforts for strengthening or reforming health financing systems occur may be different with the SDGs declaration. This is because health financing reform is a political process, and SDGs is a political declaration, with UHC one of its targets. Effective, efficient and equitable health financing systems are critical for achievement of $\operatorname{UHC}(27,28)$. Therefore, given the overarching importance of UHC as an SDG target, it is likely that the declaration has influenced health financing reforms and if so, the effects should be noticeable in changes to organization and management of subfunctions of revenue collection, pooling, purchasing, and policies on benefits and rationing. However, there are no systematic studies documenting how the declaration has influenced health financing towards UHC. Particular country experiences of how SDGs declaration has influenced health financing reforms for UHC can provide valuable lessons to stakeholders attempting to do the same in their 
countries. Given that the SDGs declaration is a relatively recent event, this review shall also provide insights on the methodological and empirical gaps in studies that explores its influence on health system financing reforms for UHC. In addition, this study will contribute broadly to the call by the World Health Organization (WHO) in 2013 of the need for evidence based research to inform initiatives that advance UHC (29).

\section{Methods}

\section{Study design, review questions and definitions}

We propose a systematic review of literature and will report on how SDGs declaration has influenced health financing reforms for UHC. Systemic review has a lower risk of bias in information synthesis compared to other review approaches (30). This protocol has been registered on the PROSPERO database (ref: CRD42020194090). PROSPERO was searched to ensure a similar systematic review study protocol has not been registered.

For this review, health financing reforms for UHC is conceptualized to refer to changes in policy objectives, strategies, or in arrangement and management of health financing system sub-functions of revenue collection, pooling, purchasing, and benefits design and rationing towards efficient and equitable system (31). These reforms should have linkage or can be attributed to global discussion on sustainable development Agenda or SDGs declaration.

In line with the preferred reporting items for systematic review and meta-analysis protocols (PRISMA-P) guideline (32), we have defined the review questions; we shall also identify, select and appraise the documents, abstract and synthesize data, and interpret the results. The review questions are: - a) How has SDGs declaration influenced the processes of health financing reform for UHC at national level? b) What dimensions of health financing system have been influenced by SDGs declaration? and c) How have Ministries of Health and other stakeholders used SDGs declaration so far to influence health financing reforms for UHC?

\section{Criteria for considering studies for this review}

All study designs will be considered for the review to account for the complex nature of health financing reforms. Specifically, we will include randomized and non-randomized studies, evaluation studies, policy analyses, stakeholder analyses, and peer-reviewed case studies and commentaries. Proposals and studies published in abstracts only will be excluded.

The inclusion criteria will be based on population, intervention (exposure) and outcomes (PEO) elements (33). That is, the document to be included for review: (i) is on health systems reforms, or other government reforms where health financing is a part of the reform process, or is specifically on health financing reforms, and is published from 2012 onwards i.e. studies conducted after the 2012 Rio de Janeiro United Nations Conference on Sustainable Development, where the process of developing SDGs 
was initiated. They will form the study population; (ii) describes how sustainable development goals declaration has influenced reforms in health systems financing for UHC as the intervention; and (iii) reports on changes to at least one of the following health financing dimensions of management or organization i.e. revenue collection, pooling, purchasing and policy on benefits design and rationing as the outcomes.

\section{Search methods for identification of studies}

Databases search: A comprehensive electronic search will be conducted using indexed and free text words in the following databases: Ovid Medline, PubMed, EBSCO, Scopus, Web of Science. Previous experience has demonstrated to us that searching 5 databases on such a topic is adequate and the process of searching additional databases does not usually yield new documents. In searching the electronic databases, we shall combine various conceptual terms for "sustainable development goals" and "health financing". We will use the Boolean operators 'OR' to combine the terms within each concept and 'AND' to combine the two concepts. We will not use search filter for study type, language (we will use the google translate for non-English articles), country or geographical area in order to find as many studies on the topic as possible. The search strategy will be validated by a librarian.

Other searches: In addition, we will screen the reference lists of included studies and contact the authors of relevant articles for further information or additional material as necessary; and conduct manual search for relevant articles from publications on websites of WHO, journals (health system reforms, health policy and planning, UHC2030 partnership), and from search engine google scholar. This is likely to yield additional pool of relevant literature.

\section{Pilot search}

In order to gauge the viability of this review, a pilot electronic search was conducted on PubMed on $2^{\text {nd }}$ June 2020 using the search strategy described under Search methods for identification of studies above. The search string was as below: -

Search: $)(((()((()((()((()(($ Health Financing Reform*) OR (Health system* financing)) OR (Health system* financing reform*)) OR (Health financing system*)) OR (Health financing system* reform*)) OR (Financing health system*)) OR (Financing healthcare)) OR (Financing health care)) OR (Health system* reform*)) OR (Health care reform*)) OR (Healthcare reform*)) OR (Health reform*)) OR (Reforming health financing)) OR (Reforming health system* financing)) OR (Reforming health care financing)) OR (Reforming healthcare financing)) OR (Reforming health)) OR (Reforming healthcare)) OR (Reforming health care)) OR (Reforming health system*)) OR (Reforming healthcare system*)) OR (Reforming health care system*)) OR (Health financing)) AND $\left(\left(\left(\left(\left(()\left(\left(\left(\left(\right.\right.\right.\right.\right.\right.\right.\right.\right.$ sustainable development goal*) OR $\left(\right.$ SDG*$\left.\left.^{*}\right)\right)$ OR (Sustainable development goal*3)) OR (SDG 3)) OR (UN development blueprint)) OR (United Nations development blueprint)) OR (global development agenda)) OR (2030 development agenda)) OR (2030 agenda)) OR (post millennium development goal*)) OR (Post-MDG*)) OR (Post MDG agenda)) Filters: in the last 5 years, Humans 
The search yielded 692 potential articles and from initial screening of titles and abstracts 69 articles were considered potentially eligible. Of the 69 titles and abstracts, 1 was in Chinese and 1 in Spanish, the rest were in English. Of the 69 articles potentially eligible, we were able to retrieve 68 articles, the exception being the one published in Chinese. The article in Spanish was translated using google scholar. After review of full length articles of the 68 articles, 2 articles were found to meet our eligibility criteria (Table 1).

Table 1: Eligible articles for review

\begin{tabular}{|lcl|}
\hline Author & Year & Country/setting \\
\hline Agustina et al(34) & 2019 & Indonesia \\
\hline Wang YP and Zhou XN(35) & 2020 & China \\
\hline
\end{tabular}

This pilot search process has indicated the potential availability of relevant literature for review.

\section{Study selection}

All retrieved articles will initially be exported to EndNote software X9 (36), where duplicates shall be removed. After removing duplicates, the EndNote library will be shared between two reviewers where the selection process will be conducted in two phases. Firstly, the two reviewers will screen the titles and abstracts of identified articles independently for potential eligibility and retrieve the full text of articles judged as potentially eligible. Secondly, full text retrieved will be screened by the two reviewers for eligibility using a standardized screening form (attachment 1 ). Disagreements will be resolved by discussion or with the help of a third reviewer.

Reviewers have undertaken a calibration exercise and refined the eligibility screening tool using results from a pilot search conducted on PubMed aforementioned. This calibration exercise has been undertaken to facilitate uniform application of eligibility criteria across reviewers. This will enhance the validity of our process and minimize bias in identification and selection documents for review. In order to improve transparency of the review process we will depict the overall selection process of documents and reasons for inclusion and exclusion in a flow chart in line with the PRISMA statement (37). The full texts of all relevant studies found to meet the inclusion criteria will be retained for the final synthesis (38). For articles that will fail to meet the inclusion criteria at the full-text screening phase, reasons for their exclusion from analysis will be documented.

\section{Data extraction}

Two reviewers will abstract data from eligible articles independently using standardized data abstraction form adapted from the Joanna Briggs Institute (JBI) data abstraction format (Attachment 2) (39). A calibration exercise will be conducted on a randomly chosen sample to ensure adequate agreement, and disagreement will be resolved by consensus or with the help of a third reviewer if consensus is not 
reached. Study characteristics to be extracted will include: the bibliographic details of study (study title, author, year of publication), objectives (purpose of the study), study design, setting (country); influence of SDGs declaration on health financing reforms; the dimension or aspect of health financing system reformed; and how Ministries of Health and other stakeholders have used SDGs declaration to drive health financing reforms for UHC.

\section{Appraisal of studies}

We shall not carry out assessment of risk of bias for the included articles for this review. This is because, existing tools for critically appraising of studies for reviews is still contested and have strong limitations, especially for qualitative studies (40). However, we shall assess the quality of the included studies.

Retrieved articles eligible for inclusion will undergo a quality assessment process during the synthesis of results. The quality appraisals will be conducted using the Joanna Briggs Institute's Critical Appraisal Checklists (see attachment 3 , an example of the JBI checklist for qualitative studies). Two reviewers will conduct the quality appraisals, where there are disagreements, a third reviewer will be engaged and discussions among the three reviewers will be used to resolve the differences. The results will help us to determine if studies included are consistent with the standard quality appraisal for articles reporting on the type of study. Quality assessment will also be used for judging the relative contribution of each study to the development of explanations and relationships between SDGs declaration and health financing reforms. It has been noted that poorer quality studies tend to contribute less to the synthesis and therefore, the synthesis becomes "weighted" towards better-quality studies (40).

\section{Synthesis and Interpretation of results}

We shall use NVIVo and a thematic framework (Attachment 4) to facilitate analysis. Thematic framework synthesis is a qualitative approach that involves selecting, recording and categorizing key issues and themes (41). For each article, the process will involve familiarization with information, identification, recording, categorization and interpretation of the influence of SDGs on health financing reforms, and how Ministries of Health and other stakeholders have used the SDGs to advance health financing reforms towards UHC. On the effects of the reform occasioned by SDGs declaration on health financing system, we will use Kutzin's conceptual framework for analyzing health financing systems (8). In using Kutzin's framework we shall focus on changes in the organization and/or management of functions of revenue collection, pooling, purchasing and policy on benefits. The results on the three review questions will be presented narratively.

\section{Discussion}

Our reporting will be aligned to the PRISMA guidelines. The report will be shared with relevant stakeholders through workshop presentations, policy briefs and journal publication. We anticipate a limitation related to finding adequate number of studies reporting on reforms in health financing in countries that have been driven by SDGs declaration. The reform processes usually take time, the SDGs 
declaration was only effected towards the end of 2015 . We shall address this by not restricting search by country, language or study design, and searching gray literature as described under methods. Despite this anticipated limitation, the proposed review will still be a valuable addition to the field of health policy and systems research as it will be the first systemic review exploring the linkage between SDGs declaration and health financing reforms at the national level. The review will also provide information on the methodological and/or empirical gaps in the area.

\section{Abbreviations}

MDGs: Millennium Development Goals; NHIS: National Health Insurance Schemes; PEO: population, exposure (intervention) and outcomes; PRISMA-P: preferred reporting items for systematic review and meta-analysis protocols; SDGs: Sustainable Development Goals; UHC: Universal Health Coverage; UN: United Nations; WHO: World Health Organization

\section{Declarations}

\section{Ethical Approval and Consent to Participate}

As this is systematic review of literature, this section is not applicable

\section{Consent for publication}

Not applicable

\section{Availability of Data and Materials}

Data sharing not applicable to this article as no datasets have been generated or analyzed at this point

\section{Competing interests}

The authors declare that they have no competing interests

\section{Funding}

This work has been funded by the authors

\section{Authors Contributions}

WDO, FS, and $\mathrm{CH}$ participated in discussing and developing the idea into a concept. WDO wrote the initial protocol, while FS and $\mathrm{CH}$ advised on the search strategy. FS and $\mathrm{CH}$ appraised the draft protocol, reviewed and approved final version for publication. All authors read and approved the final manuscript.

\section{Acknowledgements}

Ann Masese for conducting the pilot search 


\section{Authors Information}

WDO is a health policy and financing associate at the African Centre for Health Systems Development; a health policy think-tank based in Uganda. WDO is also a doctorate student at School of Health Systems and Public Health, University of Pretoria. FS is Lecturer at the School of Health Systems and Public Health, University of Pretoria and President of College of Medicine of South Africa. CH has PhD in Health Economics and Policy and is he a Research Director at the South Africa Human Sciences Research Council.

\section{References}

1. Sustainable Development Solutions Network. About the SDGs New York: Sustainable Development Solutions Network; [2019 Jun 20]. Available from: http://unsdsn.org/what-we-do/sustainabledevelopment-goals/about-the-sdgs/.

2. UN Department for Economic and Social Affairs, editor The Addis Ababa Action Agenda: financing sustainable development and developing sustainable finance. Third international conference on financing for developement; 2015; Addis Ababa. Addis Ababa2015.

3. NGO Committee on Education. UN Documents Cooperation Circles gathering a body of global agreements-agenda 21: financial resources and mechanisms 1992 [2019 May 12]. Available from: http://www.un-documents.net/a21-33.htm.

4. United Nations. World Economic and Social Survey 2014/2015: MDG lessons for post-2015 [2019 April 20]. Available from: https://wess.un.org/archive/2015/index.html.

5. United Nations. About the sustainable development goals [Available from: https://www.un.org/sustainabledevelopment/sustainable-development-goals/.

6. United Nations. Resolution adopted by the General Assembly on 25 September 2015. 2015. Report No.: 9780874216561.

7. WHO. World health report 2013: Research for universal health coverage. Luxembourg; 2013. Report No.: 92415645989789241564595.

8. Kutzin J. Health financing for universal coverage and health system performance: concepts and implications for policy. Bull World Health Organ. 2013;91(8):602-11.

9. Schieber G, Baeza C, Kress D, Maier M. Financing Health Systems in the 21st Century. In: Jamison DT, Breman JG, Measham AR, Alleyne G, Claeson M, Evans BD, et al., editors. Disease Control Priorities in Developing Countries. 2nd ed2001.

10. WHO. Everybody business: strengthening health systems to improve health outcomes: WHO's framework for action. Geneva2007.

11. Kruk ME, Gage AD, Arsenault C, Jordan K, Leslie HH, Roder-DeWan S, et al. High-quality health systems in the Sustainable Development Goals era: time for a revolution. Lancet Glob Health. 2018;6(11):e1196-e252. 
12. Mclntyre D, Kutzin J. Health financing country diagnostic: a foundation for national strategy development. Geneva: WHO; 2016.

13. Ottersen T, Elovainio R, Evans D, McCoy D, McIntyre D, Meheus F, et al. Towards a coherent global framework for health financing: Recommendations and recent developments. Health Economics, Policy and Law. 2017;12:285-96.

14. Kutzin J, Sparkes SP. Health systems strengthening, universal health coverage, health security and resilience. Bull World Health Organ. 2016;94(1):2.

15. McIntyre D, Garshong B, Mtei G, Meheus F, Thiede M, Akazili J, et al. Beyond fragmentation and towards universal coverage: insights from Ghana, South Africa and the United Republic of Tanzania. Bull World Health Organ. 2008;86(11):871-6.

16. Abuya T, Maina T, Chuma J. Historical account of the national health insurance formulation in Kenya: experiences from the past decade. BMC Health Serv Res. 2015;15:56.

17. ECSA-HC. Experince of Kenya, Uganda and Tanzania with development of national health insurance schemes. 2014.

18. Achoki T, Lesego A. Implementing Health Financing Reforms in Africa: Perspectives of Health System Stewards. Ann Glob Health. 2016;82(5):903-11.

19. Bayarsaikhan D, Musango L. Health financing issues and reforms in Africa. International Journal of Healthcare. 2016;2(2).

20. Surender R, Van Niekerk R, Hannah B, Allan L, Shung-King M. The drive for universal healthcare in South Africa: views from private general practitioners. Health Policy Plan. 2015;30(6):759-67.

21. Thomas S, Gilson L. Actor management in the development of health financing reform: health insurance in South Africa, 1994-1999. Health Policy Plan. 2004;19(5):279-91.

22. Barasa E, Nguhiu P, Mclntyre D. Measuring progress towards Sustainable Development Goal 3.8 on universal health coverage in Kenya. BMJ Glob Health. 2018;3(3):e000904.

23. Chitah BM, Chansa C, Kaonga O, Workie NW. Myriad of Health Care Financing Reforms in Zambia: Have the Poor Benefited? Health Syst Reform. 2018;4(4):313-23.

24. Carrin G, James C, Adelhardt M, Doetinchem O, Eriki P, Hassan M, et al. Health financing reform in Kenya - assessing the social health insurance proposal. S Afr Med J. 2007;97(2):130-5.

25. Basaza RK, O’Connell TS, Chapčáková I. Players and processes behind the national health insurance scheme: a case study of Uganda. BMC Health Services Research. 2013;13(1):357.

26. Aantjes C, Quinlan T, Bunders J. Towards universal health coverage in Zambia: impediments and opportunities. Development in Practice. 2016;26(3):298-307.

27. WHO. UN Sustainable Development Summit 20152015 [Available from: https://www.who.int/mediacentre/events/meetings/2015/un-sustainable-development-summit/en/.

28. Kieny MP, Bekedam H, Dovlo D, Fitzgerald J, Habicht J, Harrison G, et al. Strengthening health systems for universal health coverage and sustainable development. Bull World Health Organ. 2017. 29. WHO. Research for universal health coverage: World health report 2013. Geneva: WHO; 2013. 
30. Greenhalgh T. Papers that summarise other papers (systematic reviews and meta-analyses). Bmj. 1997;315(7109):672-5.

31. WHO. Implementing Health Financing Reform: Lessons from countries in transition. Observatory Studies Series. 2010;21:45.

32. Moher D, Shamseer L, Clarke M, Ghersi D, Liberati A, Petticrew $M$, et al. Preferred reporting items for systematic review and meta-analysis protocols (PRISMA-P) 2015 statement. Syst Rev. 2015;4(1):1.

33. Bettany-Saltikov J. Learning how to undertake a systematic review: part 1. Nurs Stand. 2010;24(50):47-55; quiz 6.

34. Agustina R, Dartanto T, Sitompul R, Susiloretni KA, Suparmi, Achadi EL, et al. Universal health coverage in Indonesia: concept, progress, and challenges. Lancet. 2019;393(10166):75-102.

35. Wang YP, Zhou XN. The year 2020, a milestone in breaking the vicious cycle of poverty and illness in China. Infect Dis Poverty. 2020;9(1):11.

36. Endnote. [Available from: http://www.endnote.com/.

37. Moher D, Liberati A, Tetzlaff J, Altman D. Preferred reporting items for systematic reviews and metaanalyses: the PRISMA statement. BMJ. 2009;339(b2535).

38. Dixon-Woods M. Using framework-based synthesis for conducting reviews of qualitative studies. BMC Med. 2011;9:39.

39. Joanna Briggs Institute. BI Reviewer's Manual 2020 [Available from: https://wiki.joannabriggs.org/display/MANUAL/JBI+Reviewer\%27s+Manual.

40. Atkins S, Lewin S, Smith H, Engel M, Fretheim A, Volmink J. Conducting a meta-ethnography of qualitative literature: Lessons learnt. BMC Medical Research Methodology. 2008;8(1):21.

41. Srivastava A, Thomson SB. Framework analysis: A qualitative methodology for applied policy research. JOAAG. 2009(4):8.

\section{Supplementary Files}

This is a list of supplementary files associated with this preprint. Click to download.

- Attachment1Screeningandeligibiltytool.docx

- Attachment1Screeningandeligibiltytool.docx

- Attachment2.DataAbstrationtool.docx

- Attachment2.DataAbstrationtool.docx

- Attachment3.Criticalappraisalchecklistdocx.docx

- Attachment3.Criticalappraisalchecklistdocx.docx

- Attachment4.Thematicanalysisframework.docx

- Attachment4.Thematicanalysisframework.docx 